\title{
Total aortic arch replacement with frozen elephant trunk (Thoraflex)
}

\author{
Luca Di Marco, Davide Pacini, Giacomo Murana, Carlo Mariani, Ciro Amodio, Roberto Di Bartolomeo \\ Cardiac Surgery Unit, Cardio-Thoraco-Vascular Department, S. Orsola Hospital, University of Bologna, Bologna, Italy \\ Correspondence to: Luca Di Marco, MD, PhD. Cardiac Surgery Unit, Cardio-Thoracic-Vascular Department, S. Orsola Hospital, University of \\ Bologna, Bologna, Italy. Email: luca.dimarco@aosp.bo.it.
}

Submitted Jan 30, 2018. Accepted for publication Feb 27, 2018.

doi: 10.21037/acs.2018.04.05

View this article at: http://dx.doi.org/10.21037/acs.2018.04.05

\section{Introduction}

The Thoraflex hybrid prosthesis represents the latest evolution of the classic frozen elephant trunk (FET) (Figure 1A,B) prosthesis. It provides a multi-branched, arch hybrid graft that potentially allows treating (for specific indications and in a single surgical step), complex lesions of the thoracic aorta by combining endovascular treatment with conventional surgery (1).

Our indications for the use of the FET procedure are chronic thoracic aortic dissection, acute type A aortic dissection (with an intimal tear or reentries in the distal arch), acute and chronic type B dissections when endovascular treatment is contraindicated and chronic aneurysms of the distal arch and proximal descending thoracic aorta. In this manuscript we show our FET technique using the Vascutek Thoraflex hybrid prosthesis.

\section{Clinical vignette}

A young 23-year-old man was referred to our hospital for a pseudo-aneurysmal thoracic aortic lesion, caused by trauma sustained during skiing. Immediately after the trauma, the patient was transported to a peripheral hospital because of thoracic and head injuries with loss of consciousness. A fullbody CT angiogram was performed and, while brain scans resulted to be negative, the thoraco-abdominal aorta scan showed a lesion of the thoracic aortic isthmus with a semicircumferential pseudo-aneurysm (Figure $2 A$ ).

After multidisciplinary evaluation, considering both the risks of an endovascular procedure performed in an emergency setting (which would necessarily imply an overstenting of the subclavian artery without carotidsubclavian by pass due to a very short proximal neck between carotid artery and subclavian artery), and the absence of signs of "impending rupture", a conservative approach was considered most appropriate. Decision was taken to postpone surgery and repeat a CT angiogram in the following few days.

During the following days, the arterial pressure remained stable with some recurrence of chest pain which resolved with the administration of analgesia. Three days later, the repeat CT angiogram showed a stable picture compared with the previous one.

However, in light of the repeated pain episodes and after a further aortic team evaluation, the patient was scheduled for a single-step thoracic aorta replacement by placing a Thoraflex hybrid prosthesis. This video focuses on the single-step procedure showing our surgical technique for FET procedure.

\section{Surgical technique}

Surgical approach consisted of a full median sternotomy and, after systemic heparinization, cardiopulmonary bypass $(\mathrm{CPB})$ was established through the proximal ascending aorta and right atrium. A left ventricle vent was placed into the right superior pulmonary vein. Cooling of the patient was initiated with a target nasopharyngeal temperature of $26^{\circ} \mathrm{C}$. The ascending aorta was clamped and cardioplegic arrest was achieved with infusion of cold crystalloid cardioplegia using the modified Bretschneider solution (Custodiol, Koehler Chemie, Alsbach-Haenlein, Germany).

Our protocol for brain protection with antegrade selective cerebral perfusion (ASCP) according with Kazui's technique has already been described (2-4). When the nasopharyngeal temperature of $26^{\circ} \mathrm{C}$ was reached, the pump flow was stopped, the circulatory arrest started, the supraaortic vessels were gently clamped while the ascending 

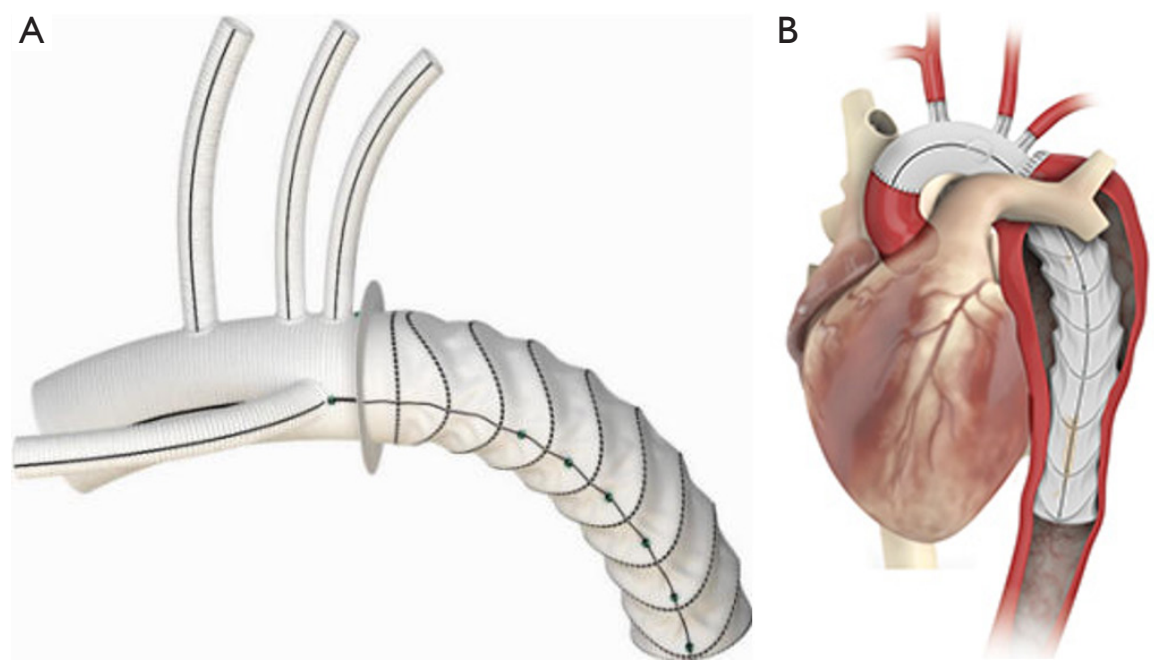

Figure 1 The Vascutek Thoraflex hybrid stent-graft (A) and its clinical application (B).
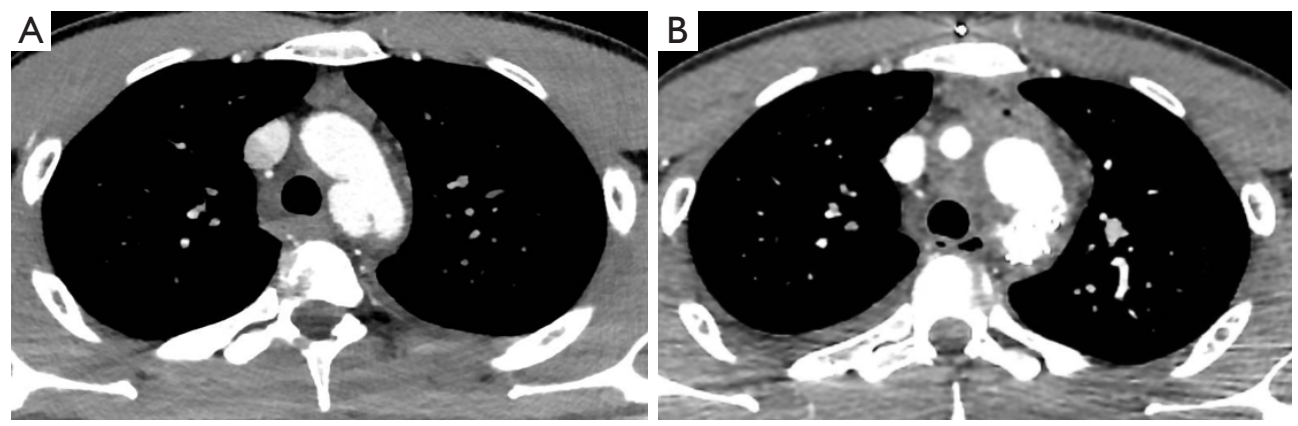

Figure 2 CT angiogram images showing preoperative lesion (A) and postoperative result (B). CT, computed tomography.

aortic clamp and the aortic cannula were removed and the aortic arch opened. An additional cardiotomy sucker was then introduced into the descending thoracic aorta and both the left common carotid artery and the innominate artery were selectively cannulated using a specific cannula for cerebral perfusion (RDB Cannula True Flow, MEDEUROPE, Bologna, Italy). In order to guarantee a flow rate of $10 \mathrm{~mL} / \mathrm{kg} / \mathrm{min}$, flow was uniformly distributed to the right and left cerebral hemispheres and adjusted to maintain the right radial pressure between 40 and $80 \mathrm{mmHg}$.

After the arch was completely resected and removed, the proximal thoracic descending aorta was inspected and the presence of a large intimal tear was documented at the convexity of the aortic arch at the isthmus level. The lesion was confirmed by the angioscope. The proximal descending aorta was then prepared using an external Teflon felt fixed with four internal pledgeted U-stitches. Then, the Vascutek
Thoraflex stent-graft system n. 22/24 was introduced in an antegrade fashion in the descending aorta. The sheath was pulled back and removed while the delivery system was anchored at the handle. The splitter was then removed from the device system and the release clip and wire were pulled in order to fully release the prosthesis from the delivery system.

Once the device was deployed, a circumferential anastomosis between the sewing collar and the previously prepared native aorta was performed with a $3 / 0$ polypropylene running suture to ensure that the implant was correctly sealed. The systemic perfusion was then antegradely restored through the side branch of the graft and rewarming of the patient was initiated. The third branch of the graft was then anastomosed to the left subclavian artery using a $5 / 0$ polypropylene running suture. The proximal prosthesis to prosthesis anastomosis was 


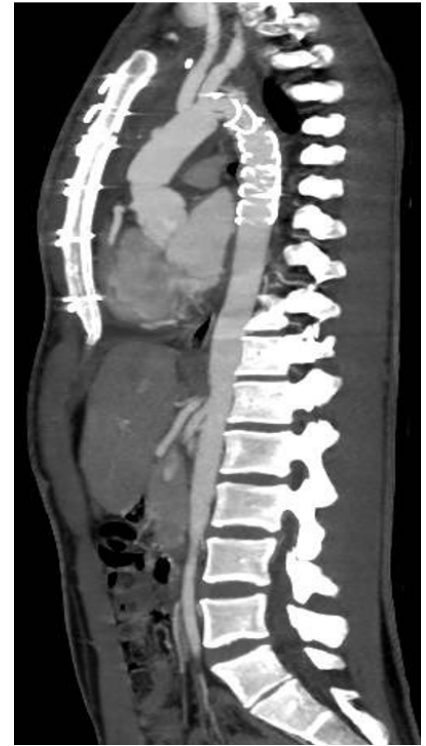

Figure 3 Postoperative CT angiogram showing normal morphology of the Thoraflex hybrid prosthesis and of the supraaortic trunks reimplantation.

then performed with a 4/0 polypropylene running suture with previous reinforcement of the proximal supracoronary ascending aorta with a double felt of heterologous pericardium. After careful deairing, the aortic clamp was removed and the myocardium reperfused. The arch reconstruction was carried out by means of left common carotid artery and innominate artery using the other two side branches of the hybrid prosthesis upon removal of ASCP cannula. When normothermia was reached, CPB was progressively weaned, with no need for inotropic support. The arterial and venous cannulae were removed and hemostasis was obtained. Finally, after positioning of drains and ventricular pacing wires, the pericardium was partially closed and the sternum resynthesized in a routine fashion.

\section{Postoperative outcome}

Two weeks after the operation the patient was discharged in good health. The postoperative period was uneventful

Cite this article as: Di Marco L, Pacini D, Murana G, Mariani C, Amodio C, Di Bartolomeo R. Total aortic arch replacement with frozen elephant trunk (Thoraflex). Ann Cardiothorac Surg 2018;7(3):451-453. doi: 10.21037/acs.2018.04.05 and the CT angiogram at postoperative day 10 showed the complete exclusion of the pseudoaneurysm of the aortic isthmus (Figure 2B) and a normal morphology of the Thoraflex hybrid prosthesis and of the re-implanted supraaortic trunks (Figure 3).

\section{Comments}

The Vascutek Thoraflex hybrid prosthesis potentially allows for treating combined lesions of the thoracic aorta in a single operation combining endovascular treatment with conventional surgery. This graft increases surgeons' options in the treatment of extensive thoracic aortic lesions.

\section{Acknowledgements}

None.

\section{Footnote}

Conflicts of Interest: The authors have no conflicts of interest to declare.

\section{References}

1. Di Bartolomeo R, Murana G, Di Marco L, et al. Frozen versus conventional elephant trunk technique: application in clinical practice. Eur J Cardiothorac Surg 2017;51:i20-8.

2. Kazui T, Inoue N, Yamada O, et al. Selective cerebral perfusion during operation for aneurysms of the aortic arch: a reassessment. Ann Thorac Surg 1992;53:109-14.

3. Pacini D, Leone A, Di Marco L, et al. Antegrade selective cerebral perfusion in thoracic aorta surgery: safety of moderate hypothermia. Eur J Cardiothorac Surg 2007;31:618-22.

4. Di Bartolomeo R, Di Marco L, Armaro A, et al. Treatment of complex disease of the thoracic aorta: the frozen elephant trunk technique with the E-vita open prosthesis. Eur J Cardiothorac Surg 2009;35:671-5; discussion 675-6. 